Canad. Math. Bull. Vol. 63 (4), 2020 pp. 813-824

http://dx.doi.org/10.4153/S0008439520000065

(c) Canadian Mathematical Society 2020 This is an Open Access article, distributed under

the terms of the Creative Commons Attribution licence (http://creativecommons.org/licenses/by/4.0/), which permits unrestricted re-use, distribution, and reproduction in any medium, provided the original work is properly cited.

\title{
Operators on Anti-dual pairs: Self-adjoint Extensions and the Strong Parrott Theorem
}

\author{
Zsigmond Tarcsay and Tamás Titkos
}

\begin{abstract}
The aim of this paper is to develop an approach to obtain self-adjoint extensions of symmetric operators acting on anti-dual pairs. The main advantage of such a result is that it can be applied for structures not carrying a Hilbert space structure or a normable topology. In fact, we will show how hermitian extensions of linear functionals of involutive algebras can be governed by means of their induced operators. As an operator theoretic application, we provide a direct generalization of Parrott's theorem on contractive completion of 2 by 2 block operator-valued matrices. To exhibit the applicability in noncommutative integration, we characterize hermitian extendibility of symmetric functionals defined on a left ideal of a $C^{*}$-algebra.
\end{abstract}

\section{Introduction}

The question whether a self-adjoint extension exists arises naturally in various situations when a partially defined (bounded or unbounded) symmetric operator is given. For classical results, we refer the reader to $[2,6,8,16]$ and the references therein; for more recent results, see, for example, [3,11]. In our previous paper [19], we developed a Krein-von Neumann type extension theory for positive operators acting on anti-dual pairs. That technique is general enough to not only overcome the lack of a Hilbert space structure, but also the lack of a normable topology. Our running example in [19] - illustrating the applicability of the general setting - came from noncommutative integration theory. Namely, we have demonstrated how functional extensions can be governed by their induced operators. The aim of this paper is to continue these investigations and to discuss the problem of self-adjoint extendibility.

Below, we briefly describe the content. Section 1 contains a short overview of concepts and earlier results that help the reader to follow the proofs. In particular, we briefly sketch the construction of the generalized Krein-von Neumann extension that serves as the basis for our treatment. In Section 2, we examine the extension problem

Received by the editors May 25, 2019; revised January 17, 2020.

Published online on Cambridge Core January 24, 2020.

The corresponding author Zs. Tarcsay was supported by DAAD-TEMPUS Cooperation Project "Harmonic Analysis and Extremal Problems" (grant no. 308015). Project no. ED 18-1-2019-0030 (Application-specific highly reliable IT solutions) has been implemented with the support provided from the National Research, Development and Innovation Fund of Hungary, financed under the Thematic Excellence Programme funding scheme.

T. Titkos was supported by the Hungarian National Research, Development and Innovation Office NKFIH (Grant No. PD128374 and Grant No. K115383), by the János Bolyai Research Scholarship of the Hungarian Academy of Sciences, and by the ÚNKP-18-4-BGE-3 New National Excellence Program of the Ministry of Human Capacities.

AMS subject classification: 47A20, 46A22, 46A20, 46K10.

Keywords: Self-adjoint operator, symmetric operator, anti-duality, self-adjoint extension, Parrott theorem, ${ }^{*}$-algebra, positive functional, hermitian functional. 
in our anti-dual pair setting. The main result, Theorem 2.1, generalizes Krein's theorem on the existence of a norm preserving self-adjoint extension of a bounded symmetric operator [10, Theorem 5.33]. Due to the lack of norm, we are going to consider extensions bounded by a fixed positive operator $A$. It will turn out that extensions preserving the $A$-bound form an operator interval. As a nice application of Theorem 2.1, in Section 3, we will generalize Yamada's recent result [21], which is an extension of the Strong Parrott Theorem [7,12]. We will close the paper by demonstrating that Theorem 2.1 on self-adjoint extensions is an effective generalization. Namely, we will see in Section 4 how this result can be applied to obtain hermitian extensions of functionals of an involutive algebra.

\section{Preliminaries}

In this section, we briefly summarize all the notions and tools to make the presentation self-contained. For more details we refer the reader to [19, Section 2 and 3]. An anti-dual pair denoted by $\langle F, E\rangle$ is a system of two complex vector spaces $E$ and $F$ intertwined by a separating sesquilinear map $\langle\cdot, \cdot\rangle: F \times E \rightarrow \mathbb{C}$, i.e., $\langle\cdot, \cdot\rangle$ is linear in its first, and conjugate linear in its second argument. Let $D$ be a linear subspace of $E$. We call a linear operator $A: D \rightarrow F$ symmetric, if $\langle A x, y\rangle=\overline{\langle A y, x\rangle}$ holds for all $x, y \in D$. In analogy with the Hilbert space case, an operator $A: D \rightarrow F$ is said to be positive if

$$
\langle A x, x\rangle \geq 0, \quad x \in D .
$$

Just as in the dual pair case, we endow $E$ and $F$ with the corresponding weak topologies $\sigma(E, F)$ (resp. $\sigma(F, E)$ ), induced by the families $\{\langle f, \cdot\rangle \mid f \in F\}$ (resp. $\{\langle\cdot, x\rangle \mid$ $x \in E\})$. Both $\sigma(E, F)$ and $\sigma(F, E)$ are locally convex Hausdorff topologies with duality properties

$$
\bar{E}^{\prime}=F \quad \text { and } \quad F^{\prime}=E .
$$

Here, $F^{\prime}$ and $\bar{E}^{\prime}$ refer to the topological dual and anti-dual space of $F$ and $E$, respectively, and the vectors $f \in F$ and $x \in E$ are identified with $\langle f, \cdot\rangle$, and $\langle\cdot, x\rangle$, respectively. We will call the anti-dual pair $\langle F, E\rangle$ weak-* sequentially complete if the topological vector space $(F, \sigma(F, E))$ is sequentially complete.

One of the useful properties of weak topologies is the following: for a topological vector space $(V, \tau)$, a linear operator $T: V \rightarrow F$ is continuous with respect to $\tau$ and $\sigma(F, E)$ if and only if the linear functionals

$$
T_{x}(v):=\langle T v, x\rangle, \quad v \in V,
$$

are continuous for every $x \in E$.

This fact and (1.1) enable us to define the adjoint (that is, the topological transpose) of a weakly continuous operator. Let $\left\langle F_{1}, E_{1}\right\rangle$ and $\left\langle F_{2}, E_{2}\right\rangle$ be anti-dual pairs and let $T: E_{1} \rightarrow F_{2}$ be a weakly continuous linear operator. Then the weakly continuous linear operator $T^{*}: E_{2} \rightarrow F_{1}$ satisfying

$$
\left\langle T x_{1}, x_{2}\right\rangle_{2}=\overline{\left\langle T^{*} x_{2}, x_{1}\right\rangle_{1}}, \quad x_{1} \in E_{1}, x_{2} \in E_{2},
$$

is called the adjoint of $T$. The set of everywhere defined weakly continuous (i.e., $\sigma(E, F)-\sigma(F, E)$ continuous) linear operators $T: E \rightarrow F$ will be denoted by $\mathscr{L}(E, F)$. In the case when $H$ and $K$ are Hilbert spaces, $\mathscr{L}(H, K)$ coincides with the set $\mathscr{B}(H, K)$ 
of bounded linear operators from $H$ to $K$. An operator $T \in \mathscr{L}(E, F)$ is called selfadjoint if $T^{*}=T$. Obviously, everywhere defined symmetric operators (and hence everywhere defined positive operators) are automatically weakly continuous and self-adjoint.

Now we proceed by recalling the construction of the Krein-von Neumann extension of a positive operator. We will use the notations of this section without further notice. For more details, see [19, Theorem 3.1 (iv) $\Rightarrow(i)]$. Let $\langle F, E\rangle$ be a $w^{*}$-sequentially complete anti-dual pair and let $A: E \supseteq \operatorname{dom} A \rightarrow F$ be a positive operator with domain $\operatorname{dom} A$. Assume further that for any $y$ in $E$, there is $M_{y} \geq 0$ such that

$$
|\langle A x, y\rangle|^{2} \leq M_{y}\langle A x, x\rangle \quad \text { for all } x \in \operatorname{dom} A .
$$

This assumption guarantees that one can build a Hilbert space $H_{A}$ by taking the Hilbert space completion of the inner product space $\left(\operatorname{ran} A,(\cdot \mid \cdot)_{A}\right)$, where

$$
\left(A x \mid A x^{\prime}\right)_{A}:=\left\langle A x, x^{\prime}\right\rangle, \quad x, x^{\prime} \in \operatorname{dom} A .
$$

Again, by (1.2), the canonical embedding operator

$$
J_{0}: H_{A} \supseteq \operatorname{ran} A \rightarrow F, \quad J_{0}(A x):=A x
$$

is weakly continuous, and thus admits a unique continuous extension $J$ to $H_{A}$ by $w^{*}$-sequentially completeness of $F$. Since $J \in \mathscr{L}\left(H_{A} ; F\right)$, we have $J^{*} \in \mathscr{L}\left(E ; H_{A}\right)$. From

$$
\left(A x^{\prime} \mid J^{*} x\right)_{A}=\left\langle J\left(A x^{\prime}\right), x\right\rangle=\left\langle A x^{\prime}, x\right\rangle=\left(A x^{\prime} \mid A x\right)_{A}, \quad x, x^{\prime} \in \operatorname{dom} A,
$$

it follows that $J^{*} x=A x$ for all $x \in \operatorname{dom} A$. As for any $x \in \operatorname{dom} A$, we have

$$
J J^{*} x=J(A x)=A x,
$$

the operator $J J^{*} \in \mathscr{L}(E, F)$ is a positive extension of $A$. We will refer to $A_{N}:=J J^{*}$ as the Krein-von Neumann extension of $A$.

We remark that the extension result above is closely related to the theory of reproducing kernels (see, for example, $[13,14,21]$ ). In fact, one can say that the operator $A: E \supseteq D \rightarrow F$ is a restriction of a reproducing kernel if and only if (1.2) holds. Finally, we mention that our assumption that $F$ be $w^{*}$-sequentially complete is weaker than the quasi-completeness imposed by Schwartz in [14].

\section{Self-adjoint Extensions of Symmetric Operators}

M. G. Krein [10] proved that every bounded symmetric Hilbert space operator possesses a norm preserving self-adjoint extension. The problem of constructing selfadjoint extensions of a symmetric operator arises in our anti-dual pair setting naturally. Since we cannot speak about norm preservation due to the lack of norm, we need to find a suitable notion to generalize Krein's theorem. Observe that the norm of a selfadjoint operator $S \in \mathscr{B}(H)$ can be expressed by means of the partial order induced by positivity. Namely, $\|S\|$ is the smallest constant $\alpha \geq 0$ such that $-\alpha I \leq S \leq \alpha I$. Based on this observation, a symmetric operator $S_{0}: E \supseteq \operatorname{dom} S_{0} \rightarrow F$ is called $A$-bounded for a fixed positive operator $A \in \mathscr{L}(E, F)$ if

$$
\left|\left\langle S_{0} x, y\right\rangle\right|^{2} \leq \alpha^{2} \cdot\langle A x, x\rangle\langle A y, y\rangle, \quad x \in \operatorname{dom} S_{0}, y \in E,
$$


holds. The smallest constant $\alpha$ is called the A-bound of $S_{0}$ and is denoted by $\alpha_{A}\left(S_{0}\right)$. We will call the extension $S \supset S_{0}$ A-bound preserving if $\alpha_{A}\left(S_{0}\right)=\alpha_{A}(S)$.

In the next theorem, which is the main result of this section, we will present a sufficient condition to guarantee for a symmetric linear operator that it possesses a self-adjoint extension. Moreover, we describe the set of all $A$-bound preserving extensions of a given symmetric operator.

Theorem 2.1 Let $\langle F, E\rangle$ be a weak-* sequentially complete anti-dual pair and let $S_{0}$ : $\operatorname{dom} S_{0} \rightarrow F$ be a symmetric operator, i.e.,

$$
\left\langle S_{0} x, y\right\rangle=\overline{\left\langle S_{0} y, x\right\rangle}, \quad x, y \in \operatorname{dom} S_{0} .
$$

Suppose that $S_{0}$ is $A$-bounded with some positive operator $A \in \mathscr{L}(E, F)$. Then there exist two distinguished self-adjoint extensions $S_{m}, S_{M} \in \mathscr{L}(E, F)$ of $S_{0}$ such that

$$
\alpha_{A}\left(S_{m}\right)=\alpha_{A}\left(S_{M}\right)=\alpha_{A}\left(S_{0}\right) .
$$

In fact, the interval $\left[S_{m}, S_{M}\right]$ consists exactly of all self-adjoint extensions $S \supset S_{0}$ such that $\alpha_{A}(S)=\alpha_{A}\left(S_{0}\right)$ :

$$
\left[S_{m}, S_{M}\right]=\left\{S \in \mathscr{L}(E, F) \mid S_{0} \subset S=S^{*}, \alpha_{A}(S)=\alpha_{A}\left(S_{0}\right)\right\} .
$$

Proof Introduce the following linear manifold

$$
\operatorname{dom} \widehat{S}_{0}:=\left\{A x \mid x \in \operatorname{dom} S_{0}\right\} \subseteq H_{A},
$$

and fix an $x \in \operatorname{dom} S_{0}$. Let us define the conjugate linear functional $f_{x}$ as

$$
f_{x}: \operatorname{ran} A \subseteq H_{A} \rightarrow \mathbb{C}, \quad f_{x}(A y):=\left\langle S_{0} x, y\right\rangle, \quad y \in E .
$$

Observe that $f_{x}$ is continuous, because

$$
\left|f_{x}(A y)\right|^{2} \leq \alpha^{2} \cdot\langle A x, x\rangle, \quad y \in E,(A y \mid A y)_{A} \leq 1
$$

holds for some $\alpha$ by $A$-boundedness. According to the Riesz representation theorem, there exists a unique representing vector $\zeta_{x} \in H_{A}$ such that

$$
\left\langle S_{0} x, y\right\rangle=\left(\zeta_{x} \mid A y\right)_{A}, \quad y \in E .
$$

If $A x=A x^{\prime}$ for some $x, x^{\prime}$ in $\operatorname{dom} S_{0}$, then $f_{x}=f_{x^{\prime}}$, and thus $\zeta_{x}=\zeta_{x^{\prime}}$. Therefore, the mapping $\widehat{S}_{0}: \operatorname{dom} \widehat{S}_{0} \rightarrow H_{A}$,

$$
\widehat{S}_{0}(A x):=\zeta_{x}
$$

is well defined and linear. By (2.2), we have

$$
\left(\widehat{S}_{0}(A x) \mid \widehat{S}_{0}(A x)\right)_{A} \leq \alpha^{2} \cdot(A x \mid A x)_{A}, \quad x \in \operatorname{dom} S_{0},
$$

whence we infer that $\widehat{S}_{0}$ is bounded with $\left\|\widehat{S}_{0}\right\|=\alpha_{A}\left(S_{0}\right)$. For $x, y$ in $\operatorname{dom} S_{0}$,

$$
\left(\widehat{S}_{0}(A x) \mid A y\right)_{A}=\left\langle S_{0} x, y\right\rangle=\overline{\left\langle S_{0} y, x\right\rangle}=\left(A x \mid \widehat{S}_{0}(A y)\right)_{A},
$$

hence $\widehat{S}_{0}$ is symmetric. Introduce the operators

$$
\widehat{T}_{m}:=\left\|\widehat{S}_{0}\right\|+\widehat{S}_{0}, \quad \widehat{T}_{M}:=\left\|\widehat{S}_{0}\right\|-\widehat{S}_{0} .
$$


Clearly, $\widehat{T}_{m}$ and $\widehat{T}_{M}$ are both positive operators on dom $\widehat{S}_{0}$. Furthermore, we have for all $h \in \operatorname{dom} \widehat{S}_{0}$ that

$$
\left\|\widehat{T}_{m} h\right\|_{A}^{2}=\left\|\widehat{S}_{0} h\right\|_{A}^{2}+2\left(\widehat{S}_{0} h \mid h\right)_{A}+\left\|\widehat{S}_{0}\right\|^{2}\|h\|_{A}^{2} \leq 2\left\|\widehat{S}_{0}\right\|\left(\widehat{T}_{m} h \mid h\right)_{A},
$$

and similarly,

$$
\left\|\widehat{T}_{M} h\right\|_{A}^{2} \leq 2\left\|\widehat{S}_{0}\right\|\left(\widehat{T}_{M} h \mid h\right)_{A} .
$$

By [19, Theorem 4.2], there exist two minimal positive extensions $\widehat{A}_{m}, \widehat{A}_{M} \in \mathscr{B}\left(H_{A}\right)$ of $\widehat{T}_{m}$ and $\widehat{T}_{M}$, respectively. Note also that

$$
\left\|\widehat{A}_{M} k\right\|_{A}^{2} \leq 2\left\|\widehat{S}_{0}\right\|\left(\widehat{A}_{M} k \mid k\right)_{A} \quad \text { and } \quad\left\|\widehat{A}_{M} k\right\|_{A}^{2} \leq 2\left\|\widehat{S}_{0}\right\|\left(\widehat{A}_{M} k \mid k\right)_{A}
$$

hold for all $k \in H_{A}$. Now set

$$
\widehat{S}_{m}:=\widehat{A}_{m}-\left\|\widehat{S}_{0}\right\| \quad \text { and } \quad \widehat{S}_{M}:=\left\|\widehat{S}_{0}\right\|-\widehat{A}_{M} .
$$

Clearly, $\widehat{S}_{m}, \widehat{S}_{M} \in \mathscr{B}\left(H_{A}\right)$ are both self-adjoint extensions of $\widehat{S}_{0}$. For $k \in H_{A}$,

$$
\left\|\widehat{S}_{m} k\right\|_{A}^{2}=\left\|\widehat{T}_{m} k\right\|_{A}^{2}-2\left\|\widehat{S}_{0}\right\|^{2}\left(\widehat{T}_{m} k \mid k\right)_{A}+\left\|\widehat{S}_{0}\right\|\|k\|_{A}^{2} \leq\left\|\widehat{S}_{0}\right\|\|k\|_{A}^{2},
$$

and therefore $\left\|\widehat{S}_{m}\right\|=\left\|\widehat{S}_{0}\right\|=\alpha_{A}\left(S_{0}\right)$. Similarly, $\left\|\widehat{S}_{M}\right\|=\alpha_{A}\left(S_{0}\right)$. Letting

$$
S_{m}:=J \widehat{S}_{m} J^{*} \quad \text { and } \quad S_{M}:=J \widehat{S}_{M} J^{*} \text {, }
$$

we conclude that $S_{m}, S_{M} \in \mathscr{L}(E, F)$ are self-adjoint operators such that

$$
\alpha_{A}\left(S_{m}\right)=\alpha_{A}\left(S_{M}\right)=\alpha_{A}\left(S_{0}\right) .
$$

Finally, for $x \in \operatorname{dom} S_{0}$ and $y \in E$,

$$
\left\langle S_{m} x, y\right\rangle=\left(\widehat{S}_{m}(A x) \mid A y\right)_{A}=\left(\widehat{S}_{0}(A x) \mid A y\right)_{A}=\left\langle S_{0} x, y\right\rangle,
$$

hence $S_{0} \subset S_{m}$. A similar calculation shows that $S_{0} \subset S_{M}$ holds as well.

To prove (2.1) let $S \in\left[S_{m}, S_{M}\right]$ and take $x \in \operatorname{dom} S_{0}$ and $y \in E$. Since $S-S_{m} \geq 0$ it follows that

$$
\begin{aligned}
\left|\left\langle\left(S-S_{m}\right) x, y\right\rangle\right|^{2} & \leq\left\langle\left(S-S_{m}\right) x, x\right\rangle\left\langle\left(S-S_{m}\right) y, y\right\rangle \\
& \leq\left\langle\left(S_{M}-S_{m}\right) x, x\right\rangle\left\langle\left(S-S_{m}\right) y, y\right\rangle=0,
\end{aligned}
$$

hence $S x=S_{m} x=S_{0} x$, that is, $S_{0} \subset S$. On the other hand $S_{m} \leq S \leq S_{M}$ implies that

$$
\begin{aligned}
|\langle S x, y\rangle| & \leq\left\langle\left(S-S_{m}\right) x, x\right\rangle^{1 / 2}\left\langle\left(S-S_{m}\right) y, y\right\rangle^{1 / 2}+\left|\left\langle S_{m} x, y\right\rangle\right| \\
& \leq 3 \alpha_{A}\left(S_{0}\right)\langle A x, x\rangle^{1 / 2}\langle A y, y\rangle^{1 / 2},
\end{aligned}
$$

hence there is a symmetric operator $\widehat{S} \in \mathscr{B}\left(H_{A}\right)$ with $\|\widehat{S}\| \leq 3 \alpha_{A}\left(S_{0}\right)$ such that $S=J \widehat{S} J^{*}$. It is clear that $\widehat{S}_{m} \leq \widehat{S} \leq \widehat{S}_{M}$, and thus $\alpha_{A}\left(S_{0}\right)=\|\widehat{S}\|=\alpha_{A}(S)$. Assume conversely that $S_{0} \subset S$ is any self-adjoint extension such that $\alpha_{A}(S)=\alpha_{A}\left(S_{0}\right)$. Then it is clear that $\alpha_{A}\left(S_{0}\right) \pm \widehat{S}$ are bounded positive extensions of $\alpha_{A}\left(S_{0}\right) \pm \widehat{S}_{0}$, hence $\widehat{A}_{m} \leq \alpha_{A}\left(S_{0}\right)+\widehat{S}$ and $\widehat{A}_{M} \leq \alpha_{A}\left(S_{0}\right)-\widehat{S}$. Consequently, $\widehat{S}_{m} \leq \widehat{S} \leq \widehat{S}_{M}$ and also $S_{m} \leq S \leq S_{M}$. The proof is complete. 
In the following corollary, we recover the classical result of Krein on self-adjoint norm-preserving extensions.

Corollary 2.2 Let $H$ be a Hilbert space and let $S_{0}: \operatorname{dom} S_{0} \rightarrow H$ be a bounded symmetric operator. Then $S$ admits two self-adjoint norm-preserving extensions $S_{m}$, $S_{M} \in \mathscr{B}(H)$ such that the interval $\left[S_{m}, S_{M}\right]$ consists exactly of all self-adjoint normpreserving extensions of $S_{0}$ :

$$
\left[S_{m}, S_{M}\right]=\left\{S \in \mathscr{B}(H) \mid S_{0} \subset S=S^{*},\left\|S_{0}\right\|=\|S\|\right\} .
$$

If a self-adjoint operator $B \in \mathscr{B}(H)$ leaving $\operatorname{dom} S_{0}$ invariant satisfies $B S_{0} \subset S_{0} B$, then

$$
S_{m} B=B S_{m}, \quad S_{M} B=B S_{M} .
$$

Proof From the proof of the previous theorem, we see that $S_{m}=\left(S_{0}+\left\|S_{0}\right\|\right)_{N}-\left\|S_{0}\right\|$ and that $S_{M}=\left\|S_{0}\right\|-\left(\left\|S_{0}\right\|-S_{0}\right)_{N}$. Here we have

$$
B\left(\left\|S_{0}\right\|-S_{0}\right) \subset\left(\left\|S_{0}\right\|-S_{0}\right) B \quad \text { and } \quad B\left(S_{0}+\left\|S_{0}\right\|\right) \subset\left(S_{0}+\left\|S_{0}\right\|\right) B,
$$

hence $B\left(S_{0}+\left\|S_{0}\right\|\right)_{N}=\left(S_{0}+\left\|S_{0}\right\|\right)_{N} B$ and $B\left(\left\|S_{0}\right\|-S_{0}\right)_{N}=\left(\left\|S_{0}\right\|-S_{0}\right)_{N} B$ due to [19, Corollary 4.3]. This clearly gives (2.3).

\section{A Generalized Strong Parrott Theorem}

The aim of this section is to generalize Parrott's famous theorem [12] on contractive extensions of 2 by 2 block operator-valued matrices, which is one of the crucial results in extension and dilation theory. As an application, we will deduce Yamada's recent result [21, Theorem 4] on the extension of the Strong Parrott Theorem [7, 20].

Theorem 3.1 Let $\left\langle F_{1}, E_{1}\right\rangle_{1}$ and $\left\langle F_{2}, E_{2}\right\rangle_{2}$ be two $w^{*}$-sequentially complete anti-dual pairs and let $T_{1}: E_{1} \supseteq \operatorname{dom} T_{1} \rightarrow F_{2}$ and $T_{2}: E_{2} \supseteq \operatorname{dom} T_{2} \rightarrow F_{1}$ be linear operators such that

$$
\left\langle T_{1} x_{1}, x_{2}\right\rangle_{2}=\overline{\left\langle T_{2} x_{2}, x_{1}\right\rangle_{1}}, \quad x_{1} \in \operatorname{dom} T_{1}, x_{2} \in \operatorname{dom} T_{2} .
$$

Assume, furthermore, that there exist two positive operators $A_{i} \in \mathscr{L}\left(E_{i} ; F_{i}\right)$ and constants $\alpha_{i} \geq 0,(i=1,2)$ such that the following estimates hold true:

$$
\begin{array}{ll}
\left|\left\langle T_{1} x_{1}, y_{2}\right\rangle_{2}\right|^{2} \leq \alpha_{1}\left\langle A_{1} x_{1}, x_{1}\right\rangle_{1}\left\langle A_{2} y_{2}, y_{2}\right\rangle_{2}, & x_{1} \in \operatorname{dom} T_{1}, y_{2} \in E_{2}, \\
\left|\left\langle T_{2} x_{2}, y_{1}\right\rangle_{1}\right|^{2} \leq \alpha_{2}\left\langle A_{1} y_{1}, y_{1}\right\rangle_{1}\left\langle A_{2} x_{2}, x_{2}\right\rangle_{2}, & x_{2} \in \operatorname{dom} T_{2}, y_{1} \in E_{1} .
\end{array}
$$

Then there exists a $T \in \mathscr{L}\left(E_{1} ; F_{2}\right)$ such that $T_{1} \subseteq T$ and $T_{2} \subseteq T^{*}$ and that

$$
\left|\left\langle T y_{1}, y_{2}\right\rangle_{1}\right|^{2} \leq \max \left\{\alpha_{1}, \alpha_{2}\right\} \cdot\left\langle A_{1} y_{1}, y_{1}\right\rangle_{1}\left\langle A_{2} y_{2}, y_{2}\right\rangle_{2}, \quad y_{1} \in E_{1}, y_{2} \in E_{2} .
$$

Proof Let us consider the pair $\left(E_{1} \times E_{2}, F_{1} \times F_{2}\right)$ with antiduality

$$
\left[\left(f_{1}, f_{2}\right),\left(e_{1}, e_{2}\right)\right]=\left\langle f_{1}, e_{1}\right\rangle_{1}+\left\langle f_{2}, e_{2}\right\rangle_{2},
$$

and introduce the following linear operator:

$$
S_{0}: E_{1} \times E_{2} \supseteq \operatorname{dom} T_{1} \times \operatorname{dom} T_{2} \rightarrow F_{1} \times F_{2}, \quad S_{0}\left(x_{1}, x_{2}\right):=\left(T_{2} x_{2}, T_{1} x_{1}\right) .
$$


An easy calculation shows that $S_{0}$ is symmetric: indeed, for $x_{1}, z_{1} \in \operatorname{dom} T_{1}$ and $x_{2}, z_{2} \in \operatorname{dom} T_{2}$,

$$
\begin{aligned}
{\left[S_{0}\left(x_{1}, x_{2}\right),\left(z_{1}, z_{2}\right)\right] } & =\left\langle T_{2} x_{2}, z_{1}\right\rangle_{1}+\left\langle T_{1} x_{1}, z_{2}\right\rangle_{2}=\overline{\left\langle T_{1} x_{z}, z_{1}\right\rangle_{1}}+\overline{\left\langle T_{2} z_{2}, x_{1}\right\rangle_{2}} \\
& =\overline{\left[S_{0}\left(z_{1}, z_{2}\right),\left(x_{1}, x_{2}\right)\right]} .
\end{aligned}
$$

Consider the positive operator $\Lambda:=\left[\begin{array}{cc}A_{1} & 0 \\ 0 & A_{2}\end{array}\right]$ acting between $E_{1} \times E_{2}$ and $F_{1} \times F_{2}$; then

$$
\begin{aligned}
& \left|\left[S_{0}\left(x_{1}, x_{2}\right),\left(y_{1}, y_{2}\right)\right]\right| \leq\left|\left\langle T_{2} x_{2}, y_{1}\right\rangle_{1}\right|+\left|\left\langle T_{1} x_{1}, y_{2}\right\rangle_{2}\right| \\
& \leq \alpha \cdot\left(\left\langle A_{1} x_{1}, x_{1}\right\rangle_{1}^{1 / 2}\left\langle A_{2} y_{2}, y_{2}\right\rangle_{2}^{1 / 2}+\left\langle A_{1} y_{1}, y_{1}\right\rangle_{1}^{1 / 2}\left\langle A_{2} x_{2}, x_{2}\right\rangle_{2}^{1 / 2}\right) \\
& \left.\quad \leq \alpha \cdot\left[\Lambda\left(x_{1}, x_{2}\right),\left(x_{1}, x_{2}\right)\right]^{1 / 2}\left[\Lambda\left(y_{1}, y_{2}\right),\left(y_{1}, y_{2}\right)\right)\right]^{1 / 2}
\end{aligned}
$$

for $x_{i} \in \operatorname{dom} T_{i}, y_{i} \in E_{i}(i=1,2)$ and with $\alpha:=\max \left\{\sqrt{\alpha_{1}}, \sqrt{\alpha_{2}}\right\}$. This means that $S_{0}$ and $\Lambda$ fulfill all the conditions of Theorem 2.1. Hence, we conclude that there exists a self-adjoint operator $S \in \mathscr{L}\left(E_{1} \times E_{2} ; F_{1} \times F_{2}\right)$, which extends $S_{0}$ and that

$$
\left|\left[S\left(y_{1}, y_{2}\right),\left(w_{1}, w_{2}\right)\right]\right|^{2} \leq \alpha^{2} \cdot\left[\Lambda\left(y_{1}, y_{2}\right),\left(y_{1}, y_{2}\right)\right]\left[\Lambda\left(w_{1}, w_{2}\right),\left(w_{1}, w_{2}\right)\right],
$$

$y_{i}, w_{i} \in E_{i}(i=1,2)$. Let us interpret $S$ as an operator matrix of the form

$$
S=\left[\begin{array}{cc}
B_{1} & T^{*} \\
T & B_{2}
\end{array}\right],
$$

where $B_{i} \in \mathscr{L}\left(E_{i} ; F_{i}\right)$ are self-adjoint operators and $T \in \mathscr{L}\left(E_{1} ; F_{2}\right)$. We claim that $T$ possesses the desired properties. Indeed,

$$
\left\langle T x_{1}, y_{2}\right\rangle_{2}=\left[S\left(x_{1}, 0\right),\left(0, y_{2}\right)\right]=\left[S_{0}\left(x_{1}, 0\right),\left(0, y_{2}\right)\right]=\left\langle T_{1} x_{1}, y_{2}\right\rangle_{2},
$$

for $x_{1} \in \operatorname{dom} T_{1}, y_{2} \in E_{2}$, and similarly,

$$
\left\langle T^{*} x_{2}, y_{1}\right\rangle_{1}=\left[S\left(0, x_{2}\right),\left(y_{1}, 0\right)\right]=\left[S_{0}\left(0, x_{2}\right),\left(y_{1}, 0\right)\right]=\left\langle T_{2} x_{2}, y_{1}\right\rangle_{1},
$$

for $x_{2} \in \operatorname{dom} T_{2}, y_{1} \in E_{1}$, thus we conclude that $T_{1} \subset T$ and $T_{2} \subset T^{*}$. Finally,

$$
\begin{aligned}
\left|\left\langle T y_{1}, y_{2}\right\rangle_{2}\right|^{2} & =\left|\left[S\left(y_{1}, 0\right),\left(0, y_{2}\right)\right]\right|^{2} \\
& \leq \alpha^{2} \cdot\left[\Lambda\left(y_{1}, 0\right),\left(y_{1}, 0\right)\right]\left[\Lambda\left(0, y_{2}\right),\left(0, y_{2}\right)\right] \\
& =\alpha^{2} \cdot\left\langle A_{1} y_{1}, y_{1}\right\rangle_{1}\left\langle A_{2} y_{2}, y_{2}\right\rangle_{2},
\end{aligned}
$$

for $y_{i} \in E_{i}, i=1,2$, which completes the proof.

Using the generalized Parrott theorem above, we obtain a new proof for a recent result of A. Yamada [21, Theorem 4].

Corollary 3.2 Let $\left\langle F_{1}, E_{1}\right\rangle_{1},\left\langle F_{2}, E_{2}\right\rangle_{2}$ be anti-dual pairs and let $H, K$ be Hilbert spaces. For $S_{1} \in \mathscr{L}\left(E_{1}, H\right), S_{2} \in \mathscr{L}\left(E_{1}, K\right), T_{1} \in \mathscr{L}\left(H, F_{2}\right)$, and $T_{2} \in \mathscr{L}\left(K, F_{2}\right)$, the following conditions are equivalent:

(i) $T_{1} S_{1}=T_{2} S_{2}, S_{2}^{*} S_{2} \leq S_{1}^{*} S_{1}$, and $T_{1} T_{1}^{*} \leq T_{2} T_{2}^{*}$;

(ii) there exists $X \in \mathscr{B}(H, K),\|X\| \leq 1$, such that $X S_{1}=S_{2}$ and $T_{2} X=T_{1}$, i.e., $X$ makes the following diagram commutative: 


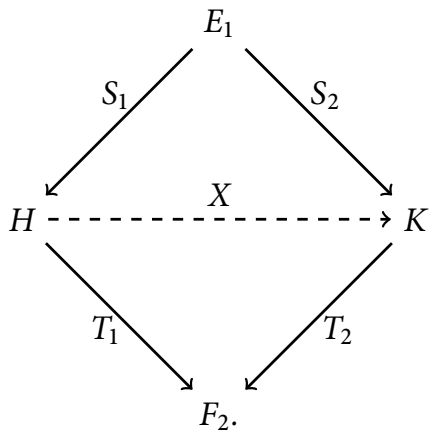

Proof Implication (ii) $\Rightarrow(\mathrm{i})$ is straightforward, so we only prove that (i) implies (ii). Consider the anti-dual pairs $(H \mid H)$ and $(K \mid K)$ and the operators

$$
X_{0}: H \supseteq \operatorname{ran} S_{1} \rightarrow K \quad \text { and } \quad X_{1}: K \supseteq \operatorname{ran} T_{2}^{*} \rightarrow H,
$$

defined by

$$
X_{0}\left(S_{1} x_{1}\right):=S_{2} x_{1}, \quad X_{1}\left(T_{2}^{*} x_{2}\right):=T_{1}^{*} x_{2} .
$$

From (i), we see that $X_{0}, X_{1}$ are well defined contractions such that

$$
\left(X_{0}\left(S_{1} x_{1}\right) \mid T_{2}^{*} x_{2}\right)=\left(S_{2} x_{1} \mid T_{2}^{*} x_{2}\right)=\left(S_{1} x_{1} \mid T_{1}^{*} x_{2}\right)=\left(S_{1} x_{1} \mid X_{1}\left(T_{2}^{*} x_{2}\right)\right),
$$

for every $x_{1} \in E_{1}$ and $x_{2} \in E_{2}$. Hence, the pair $X_{0}, X_{1}$ fulfills the conditions of Theorem 3.1 with $A_{1}=I_{H}, A_{2}=I_{K}$ and $\alpha_{1}=\alpha_{2}=1$. Consequently, there exists $X \in \mathscr{B}(H, K),\|X\| \leq 1$ such that $X_{0} \subset X, X_{1} \subset X^{*}$, and therefore $X S_{1}=S_{2}$ and $X^{*} T_{2}^{*}=T_{1}^{*}$, which yields (ii).

Yamada's work itself generalizes Parrott's theorem [12] and the Strong Parrott Theorem (see $[1,4,7])$. So we get the following classical result automatically.

Corollary 3.3 Let $H$ and $K$ be Hilbert spaces, let $H_{1} \subseteq H$ and $K_{1} \subseteq K$ be closed linear subspaces, and denote by $P_{K_{1}}$ the orthogonal projection onto $K_{1}$. For given contractions $T_{1}: H_{1} \rightarrow K$ and $T_{1}^{\prime}: H \rightarrow K_{1}$, the following conditions are equivalent:

(i) $P_{K_{1}} T_{1}=\left.T_{1}^{\prime}\right|_{H_{1}}$;

(ii) there exists a contraction $T \in \mathscr{B}(H, K)$ such that

$$
T_{1}=\left.T\right|_{H_{1}} \text { and } T_{1}^{\prime}=P_{K_{1}} T \text {. }
$$

\section{Hermitian Extensions of Linear Functionals}

Positive functionals play an important role in the representation theory of algebras. Extension of such functionals has been investigated in many different settings. For example, if $f$ is a positive linear functional defined on a closed ideal in a $C^{*}$-algebra, then $f$ always admits an extension with the same norm (see [5, II.6.4.16]). Positive functionals defined on left-ideals of the full operator algebra possessing normal extension were characterized in [18], while positive extendibility of positive functionals defined on left ideals of general * ${ }^{*}$-algebras was studied in [19]. The aim of this section is to demonstrate how our anti-dual pair setting can be used to construct hermitian extensions of linear functionals in the unital ${ }^{*}$-algebra setting. 
Let $\mathscr{A}$ be a unital ${ }^{\star}$-algebra with unit 1 . If $\mathscr{I} \subseteq \mathscr{A}$ is a left ideal, then we call a linear functional $g: \mathscr{I} \rightarrow \mathbb{C}$ symmetric if

$$
g\left(b^{*} a\right)=\overline{g\left(a^{*} b\right)}, \quad a, b \in \mathscr{I} .
$$

A linear functional $g \in \mathscr{A}^{*}$ is called hermitian if it satisfies

$$
g\left(x^{*}\right)=\overline{g(x)}, \quad x \in \mathscr{A} .
$$

It is easy to check that a linear functional $g \in \mathscr{A}^{*}$ is hermitian if and only if it is symmetric. Note that for a ${ }^{*}$-algebra without unit element, this equivalence no longer holds.

Assume that a positive linear functional $f: \mathscr{A} \rightarrow \mathbb{C}$ is given. We say that the symmetric functional $g: \mathscr{I} \rightarrow \mathbb{C}$ is $f$-bounded if

$$
\left|g\left(x^{*} a\right)\right|^{2} \leq \alpha^{2} f\left(x^{*} x\right) f\left(a^{*} a\right), \quad x \in \mathscr{A}, a \in \mathscr{I},
$$

holds for some $\alpha>0$. The $f$-bound $\alpha_{f}(g)$ is defined as the smallest constant $\alpha$ that fulfills (4.1). If $\ell: \mathscr{I} \rightarrow \mathbb{C}$ is a linear functional, then the correspondence

$$
\langle L a, x\rangle:=\ell\left(x^{*} a\right), \quad a \in \mathscr{I}, x \in \mathscr{A},
$$

defines a linear operator $L: \mathscr{I} \rightarrow \mathscr{A}^{*}$. Clearly, $L$ is positive if $\ell$ is positive and $L$ is symmetric if $\ell$ is so. Suppose now that $f \in \mathscr{A}^{*}$ is a positive functional and denote by $A$ the positive operator associated with $f$, i.e.,

$$
\langle A x, y\rangle=f\left(y^{*} x\right), \quad x, y \in \mathscr{A} .
$$

Let $H_{A}$ denote the corresponding Hilbert space, that is, $H_{A}$ is the completion of $\operatorname{ran} A$ endowed with the inner product (1.3). Observe that in that case, we have

$$
(A x \mid A y)_{A}=f\left(y^{*} x\right), \quad x, y \in \mathscr{A} .
$$

Consider the canonical embedding $J: H_{A} \rightarrow \overline{\mathscr{A}}^{*}$ in (1.4) and recall its useful properties

$$
J^{*} x=A x, \quad x \in \mathscr{A},
$$

and $J J^{*}=A$. Assume in addition that

$$
\left|f\left(y^{*} x^{*} x y\right)\right| \leq M_{x} f\left(y^{*} y\right), \quad x, y \in \mathscr{A}
$$

for some $M_{x} \geq 0$. This assures that the operators $\pi_{f}(x)$ defined by

$$
\pi_{f}(x)\left(J^{*} y\right):=J^{*}(x y), \quad y \in \mathscr{A},
$$

are continuous on $H_{A}$ by norm bound $M_{x}^{1 / 2}$. Thus, for every $x \in \mathscr{A}$, we can extend $\pi_{f}(x)$ to an element of $\mathscr{B}\left(H_{A}\right)$. It is then immediate that $\pi_{f}: \mathscr{A} \rightarrow \mathscr{B}\left(H_{A}\right)$ is a *-homomorphism such that

$$
f(x)=\left(\pi_{f}(x)\left(J^{*} 1\right) \mid J^{*} 1\right)_{A}, \quad x \in \mathscr{A} .
$$

A positive functional satisfying (4.2) (and hence (4.3)) will be called representable [15].

Theorem 4.1 Let $\mathscr{A}$ be a unital *-algebra, $\mathscr{I} \subseteq \mathscr{A}$ a left ideal and $f \in \mathscr{A}^{*}$ a representable positive functional. If $g_{0}: \mathscr{I} \rightarrow \mathbb{C}$ is an $f$-bounded symmetric functional with $f$-bound $\alpha_{f}\left(g_{0}\right)$, then there exist two distinguished $f$-bounded hermitian functionals $g_{m}, g_{M} \in \mathscr{A}^{*}$ with $f$-bound $\alpha_{f}\left(g_{m}\right)=\alpha_{f}\left(g_{M}\right)=\alpha_{f}\left(g_{0}\right)$ extending $g_{0}$. Furthermore, 
$g_{m} \leq g_{M}$ and the interval $\left[g_{m}, g_{M}\right]$ consists of all hermitian $f$-bound preserving extensions of $g_{0}$ :

$$
\left[g_{m}, g_{M}\right]=\left\{g \in \mathscr{A}^{*} \mid g_{0} \subset g=g^{*}, \alpha_{f}(g)=\alpha_{f}\left(g_{0}\right)\right\} .
$$

Proof Along the lines of the proof of Theorem 2.1, let us introduce a symmetric operator $S_{0}$ on $\operatorname{dom} S_{0}:=\left\{J^{\star} a \mid a \in \mathscr{I}\right\}$ such that

$$
\left(S_{0}\left(J^{*} a\right) \mid J^{*} x\right)_{A}=g_{0}\left(x^{*} a\right), \quad a \in \mathscr{I}, x \in \mathscr{A} .
$$

A straightforward calculation shows that $S_{0}$ : $\operatorname{dom} S_{0} \rightarrow H_{A}$ is bounded with norm $\left\|S_{0}\right\|=\alpha_{f}\left(g_{0}\right)$. For $x, y \in \mathscr{A}$ and $a \in \mathscr{I}$, we have

$$
\begin{aligned}
\left(S_{0} \pi_{f}(x)\left(J^{*} a\right) \mid J^{*} y\right)_{A} & =\left(S_{0} J^{*}(x a) \mid J^{*} y\right)_{A}=g\left(y^{*} x a\right)=\left(S_{0}\left(J^{*} a\right) \mid J^{*}\left(x^{*} y\right)\right)_{A} \\
& =\left(S_{0} J^{*} a \mid \pi_{f}\left(x^{*}\right) J^{*} y\right)_{A}=\left(\pi_{f}(x) S_{0}\left(J^{*} a\right) \mid J^{*} y\right)_{A} ;
\end{aligned}
$$

hence, we infer that dom $S_{0}$ is $\pi_{f}$-invariant and $\pi_{f}(x) S_{0} \subset S_{0} \pi_{f}(x)$ for all $x \in \mathscr{A}$. By Corollary 2.2, it follows that there exist two norm preserving self-adjoint extensions $S_{m}, S_{M} \in \mathscr{B}\left(H_{A}\right)$ of $S_{0}$ such that

$$
\pi_{f}(x) S_{m}=S_{m} \pi_{f}(x), \quad \text { and } \quad \pi_{f}(x) S_{M}=S_{M} \pi_{f}(x),
$$

whenever $x$ is self-adjoint, and hence also for every $x \in \mathscr{A}$. We claim that the functionals

$$
g_{m}(x):=\left(S_{m} J^{*} x \mid J^{*} 1\right)_{A}, \quad \text { and } \quad g_{M}(x):=\left(S_{m} J^{*} x \mid J^{*} 1\right)_{A}, \quad x \in \mathscr{A},
$$

fulfill all conditions of the statement. First, we observe that $g_{m}$ and $g_{M}$ are hermitian; indeed, by (4.4), we have for every $x \in \mathscr{A}$ that

$$
g_{m}\left(x^{*}\right)=\left(S_{m} J^{*} x^{*} \mid J^{*} 1\right)_{A}=\left(\pi_{f}\left(x^{*}\right) S_{m} J^{*} 1 \mid J^{*} 1\right)_{A}=\left(S_{m} J^{*} 1 \mid J^{*} x\right)_{A}=\overline{g_{m}(x)} .
$$

A similar argument shows that $g_{M}$ is hermitian. Next, observe that $g_{m}$ and $g_{M}$ extend $g_{0}$, because for every $a \in \mathscr{I}$,

$$
g_{m}(a)=\left(S_{m} J^{*} a \mid J^{*} 1\right)_{A}=\left(S_{0} J^{*} a \mid J^{*} 1\right)_{A}=g(a)
$$

holds, and similarly, $g_{M}(a)=g(a)$. Finally, we have

$$
\left\|S_{m}\right\|=\left\|S_{M}\right\|=\|S\|=\alpha_{f}\left(g_{0}\right),
$$

whence it follows readily that $g_{m}$ and $g_{M}$ have $f$-bound $\alpha_{f}\left(g_{0}\right)$.

Now, let $g \in \mathscr{A}^{*}$ be an arbitrary hermitian extension of $g_{0}$ having $f$-bound $\alpha_{f}\left(g_{0}\right)$. Then there is a self-adjoint operator $S \in \mathscr{B}\left(H_{A}\right),\|S\|=\alpha_{f}\left(g_{0}\right)$ such that

$$
\left(S J^{*} x \mid J^{*} y\right)_{A}=g\left(y^{*} x\right), \quad x, y \in \mathscr{A} .
$$

It is clear that $S_{0} \subset S$, and therefore, $S_{m} \leq S \leq S_{M}$, due to Corollary 2.2. As a straightforward consequence, we conclude that $g_{m} \leq g \leq g_{M}$. Suppose conversely that $g \in \mathscr{A}^{*}$ is a hermitian functional such that $g_{m} \leq g \leq g_{M}$. First, observe that $g$ is $f$-bounded as it satisfies

$$
\begin{aligned}
\left|g\left(y^{*} x\right)\right| & \leq\left(g-g_{m}\right)\left(x^{*} x\right)^{1 / 2}\left(g-g_{m}\right)\left(y^{*} y\right)^{1 / 2}+\left|g_{m}\left(y^{*} x\right)\right| \\
& \leq 3 \alpha_{f}\left(g_{0}\right) f\left(x^{*} x\right)^{1 / 2} f\left(y^{*} y\right)^{1 / 2} .
\end{aligned}
$$


Hence, there exists a self-adjoint operator $S \in \mathscr{B}\left(H_{A}\right)$, such that

$$
g\left(y^{*} x\right)=\left(J S J^{*} x \mid y\right)_{A}, \quad x, y \in \mathscr{A} .
$$

From $g_{m} \leq g \leq g_{M}$, it follows that $S_{m} \leq S \leq S_{M}$, and therefore, $S_{0} \subset S$ by Corollary 2.2. Consequently,

$$
g_{0}(a)=\left(S_{0} J^{*} a \mid J^{*} 1\right)_{A}=\left(S J^{*} a \mid J^{*} 1\right)_{A}=g(a), \quad a \in \mathscr{I} ;
$$

thus, $g_{0} \subset g$. The proof is complete.

We remark that Theorem 4.1 provides only a sufficient condition for the existence of hermitian extensions. On $C^{*}$-algebras, the statement of Theorem 4.1 may be improved in two ways; first, the condition on $f$ of being representable can be replaced by the formally weaker one of being positive. On the other hand, the existence of a dominating positive functional is both necessary and sufficient.

Corollary 4.2 Let $\mathscr{A}$ be a unital $C^{*}$-algebra and $\mathscr{I} \subseteq \mathscr{A}$ a left ideal. A linear functional $g_{0}: \mathscr{I} \rightarrow \mathbb{C}$ possesses a continuous hermitian extension $g$ if and only if $g_{0}$ is symmetric and $f$-bounded for some positive functional $f \in \mathscr{A}^{*}$.

Proof On a $C^{*}$-algebra, every positive functional $f$ is representable. If $g_{0}$ is symmetric and $f$-bounded, then $g_{0}$ has a hermitian extension $g$ that is of the form

$$
g(a)=\left(S J^{*} a \mid J^{*} 1\right), \quad a \in \mathscr{A},
$$

where $S$ is a bounded self-adjoint operator on $H_{A}$. It follows therefore that $g$ is continuous. For the converse, assume that $g \in \mathscr{A}^{*}$ is a continuous hermitian extension of $g_{0}$. Let $g=g_{+}-g_{-}$be the Hahn-Jordan decomposition of $g$, with $g_{+}, g_{-} \in \mathscr{A}^{*}$ positive functionals (see [9]). Letting $f:=g_{+}+g_{-}$, it is easy to check that $g_{0}$ is $f$-bounded with bound $\alpha_{f}\left(g_{0}\right)=4$.

Acknowledgement We thank the referees for their time and for careful reading of the manuscript. Their comments helped improve the quality of the paper immensely.

\section{References}

[1] T. Ando and T. Hara, Another approach to the strong Parrott theorem. J. Math. Anal. Appl. 171(1992), 125-130. https://doi.org/10.1016/0022-247X(92)90380-V

[2] T. Ando and K. Nishio, Positive self-adjoint extensions of positive symmetric operators. Tohoku Math. J. 22(1970), 65-75. https://doi.org/10.2748/tmj/1178242861

[3] D. Baidiuk and S. Hassi, Completion, extension, factorization, and lifting of operators. Math. Ann. 364(2016), 1415-1450. https://doi.org/10.1007/s00208-015-1261-5

[4] M. Bakonyi and H. J. Woerdeman, On the strong Parrott completion problem. Proc. Amer. Math. Soc. 117(1993), 429-433. https://doi.org/10.2307/2159179

[5] B. Blackadar, Operator algebras: theory of $C^{*}$-algebras and von Neumann algebras. Encyclopaedia of Mathematical Sciences, 122, Operator Algebras and Non-Commutative Geometry III, Springer-Verlag, Berlin, 2006. https://doi.org/10.1007/3-540-28517-2

[6] E. A. Coddington and H. S. V. de Snoo, Positive selfadjoint extensions of positive symmetric subspaces. Math. Zeitschriften 159(1978), 203-214. https://doi.org/10.1007/BF01214571

[7] C. Foias and A. Tannenbaum, A strong Parrott theorem. Proc. Amer. Math. Soc. 106(1989), 777-784. https://doi.org/10.2307/2047435

[8] S. Hassi, M. M. Malamud, and H.S.V. de Snoo, On Krein's extension theory of nonnegative operators. Math. Nachr. 274/275(2004), 40-73. https://doi.org/10.1002/mana.200310202 
[9] R. Kadison and J. Ringrose, Fundamentals of the theory of operator algebras. Vol. I. Elementary theory. Pure and Applied Mathematics, 100, Academic Press, New York, 1983.

[10] M. G. Krein, The theory of self-adjoint extensions of semi-bounded Hermitian transformations and its applications. I. Rec. Math. [Mat. Sbornik] N.S. 20(1947), no. 62, 431-495.

[11] M. M. Malamud, On some classes of extensions of sectorial operators and dual pairs of contractions. In: Recent advances in operator theory. Operator theory: advances and applications, 124, Birkhäuser, Basel, 2001, pp. 401-449.

[12] S. Parrott, On the quotient norm and the Sz.-Nagy-Foias lifting theorem. J. Functional Analysis 30(1978), 311-328. https://doi.org/10.1016/0022-1236(78)90060-5

[13] V. Paulsen and M. Raghupathi, An introduction to the theory of reproducing kernel Hilbert spaces. Cambridge Studies in Advanced Mathematics, 152, Cambridge University Press, Cambridge, 2016. https://doi.org/10.1017/CBO9781316219232

[14] L. Schwartz, Sous-espaces hilbertiens d'espaces vectoriels topologiques et noyaux associés (noyaux reproduisants). J. Analyse Math. 13(1964), 115-256. https://doi.org/10.1007/BF02786620

[15] Z. Sebestyén, On representability of linear functionals on *-algebras. Period. Math. Hungar. 15(1984), 233-239. https://doi.org/10.1007/BF02454172

[16] Z. Sebestyén, Operator extensions on Hilbert space. Acta Sci. Math. (Szeged) 57(1993), 233-248.

[17] Z. Sebestyén, Zs. Szücs, and Zs. Tarcsay, Extensions of positive operators and functionals. Linear Algebra Appl. 472(2015), 54-80. https://doi.org/10.1016/j.laa.2015.01.028

[18] Z. Sebestyén, Zs. Tarcsay, and T. Titkos, A characterization of positive normal functionals on the full operator algebra. In: The diversity and beauty of applied operator theory. Oper. Theory Adv. Appl., 268, Birkhäuser, Cham, 2018, pp. 443-447.

[19] Zs. Tarcsay and T. Titkos, Operators on anti-dual pairs: Generalized Krein-von Neumann extension. 2018. arxiv: 1810.02619

[20] D. Timotin, A note on Parrott's strong theorem. J. Math. Anal. Appl. 171(1992), 288-293. https://doi.org/10.1016/0022-247X(92)90390-Y

[21] A. Yamada, Parrott's theorem and bounded solutions of a system of operator equations. Complex Anal. Oper. Theory 11(2017), 961-976. https://doi.org/10.1007/s11785-016-0559-y

Department of Applied Analysis and Computational Mathematics, Eötvös Loránd University,

Pázmány Péter sétány 1/c., Budapest H-1117, Hungary

e-mail: tarcsay@cs.elte.hu

Alfréd Rényi Institute of Mathematics, Reáltanoda utca 13-15., Budapest H-1053, Hungary, and BBS University of Applied Sciences, Alkotmány u. 9., Budapest H-1054, Hungary

e-mail: titkos@renyi.hu 